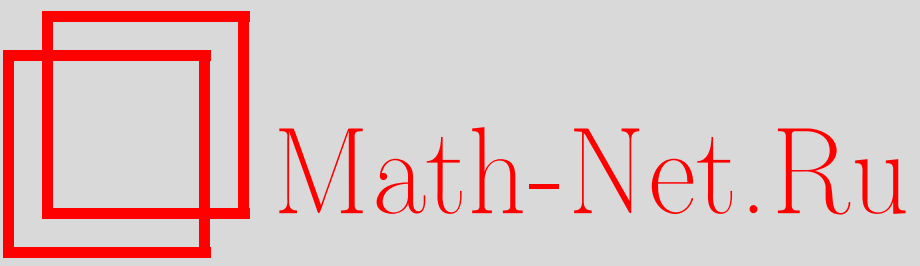

А. В. Егоров, О конечно-порожденных группах унитарных операторов, УМН, 1999, том 54, выпуск 3, 159-160

DOI: https://doi.org/10.4213/rm159

Использование Общероссийского математического портала Math-Net.Ru подразумевает, что вы прочитали и согласны с пользовательским соглашением

http://www.mathnet.ru/rus/agreement

Параметры загрузки:

IP : 54.209 .52 .79

26 апреля 2023 г., 16:31:56 


\title{
О КОНЕЧНО-ПОРОЖДЕННЫХ ГРУППАХ УНИТАРНЫХ ОПЕРАТОРОВ
}

\author{
А. В. Егоров
}

В современной алгебре вопрос о сушествовании точного линейного представления группы решается с помощью метода расщепляемых координат [1]. В предлагаемой работе рассматривается новый метод построения точных представлений, использующий понятия топологической динамики.

Пусть $G$ - конечно-порожденная группа, $K$ - компактное метризуемое пространство. Предположим, что группа $G$ действует гомеоморфизмами на компакте $K$. Напомним, что такое действие называется дистальныц.м, если для всякой пары точек $k, k^{\prime} \in K$ и произвольной последовательности элементов $g_{1}, g_{2}, \ldots, g_{i}, \ldots \in G$ пределы соответствующих последовательностей сдвигов $g_{1} k, g_{2} k, \ldots, g_{i} k, \ldots$ и $g_{1} k^{\prime}, g_{2} k^{\prime}, \ldots, g_{i} k^{\prime}, \ldots$ различны (если эти пределы существуют). Назовем действие группы $G$ на компакте $K$ действием без малых орбит, если существует точка $k \in K$ с такой окрестностью $U$, что для всякого элемента $g \in G$ найдется некоторая его степень $g^{m}$, под действием которой точка $k$ выходит за пределы окрестности $U$.

Теорема. Для того чтобы конечно-порожденная группа могла быть реализована как подгруппа группы унитарных операторов в некотором конечномерном комплексном линейном пространстве, необходимо и достаточно, чтобы она допускала эффективное дистальное действие без малых орбит на некотором компакте.

Если конечно-порожденная группа реализована как подгруппа группы унитарных операторов $U_{n}(\mathbb{C})$, то в качестве требуемого действия можно взять ее действие левыми сдвигами на $U_{n}(\mathbb{C})$. Действительно, пространство $U_{n}(\mathbb{C})$ компактно и может быть наделено левоинвариантной метрикой. Отсюда следует дистальность действия. Свойство отсутствия малых орбит следует из свойства отсутствия малых подгрупп в $U_{n}(\mathbb{C})$, так как $U_{n}(\mathbb{C})$ являтся группой Ли.

Обратно, если конечно-порожденная группа $G$ действует требуемым образом на компакте $K$, то она может быть вложена в компактную топологическую метризуемую группу $\widehat{G}$, причем это вложение обладает относительным NSS-свойством: существует окрестность единицы в $\widehat{G}$, которая не содержит подгрупп из $G$. Тогда, используя теорему Петера-Вейля и компактность группы $\widehat{G}$, можно построить достаточную систему унитарных конечномерных представлений группы $G$, состоящую из конечного числа представлений. Сумма этих представлений задает точное унитарное конечномерное представление группы $G$. Для построения вложения $G \subset \widehat{G}$ используется конструкция, связанная с действиями на банаховых пространствах. Имеет место следующее утверждение.

Лемма. Пусть $G$ - конечно-порожденная группа, әффективно почти-периодически действующая на вещественном банаховом пространстве $B$ линейными непрерывными операторами. Почти-периодичность действия означает, что замыкание $\widehat{G}_{b}$ орбиты каждого вектора $b \in B$ компактно. Тогда замыкание естественного вложсения $G$ в $\widetilde{G}=\prod_{b \in B} \widehat{G}_{b}$ является компактной топологической метризуемой группой.

Топологическое произведение $\widetilde{G}$ компактно согласно теореме Тихонова. Каждая точка этого произведения интерпретируется как отображение, которое ставит в соответствие вектору $b \in B$ некоторый вектор из замкнутой орбиты $\widehat{G}_{b}$. Композиция отображений такого вида снова происходит из некоторой точки пространства $\widetilde{G}$. Поэтому пространство $\widetilde{G}$ приобретает структуру моноида. Каждый элемент $G$ рассматривается как обратимый линейный оператор и отождествляется с некоторой точкой $\widetilde{G}$. Таким образом, мы получаем изоморфное вложение $G$ в $\widetilde{G}$. Требуемые свойства замыкания образа этого вложения доказываются с использованием теоремы Банаха-Штейнгауза.

В рассматриваемом нами случае группа $G$, действующая на компакте $K$, является подгруппой в обволакивающей полугруппе Эллиса $E(G, K)$. Обволакивающая полугруппа получается 
присоединением поточечных пределов преобразований из $G$. Ввиду дистальности действия полугруппа Эллиса оказывается группой [2]. Это обстоятельство позволяет доказать, что действие группы $G$ на компакте $K$ индуцирует дистальное по отношению к слабой сходимости действие на слабо замкнутой выпуклой оболочке левых сдвигов борелевской вероятностной меры на $E(G, K)$. Существование $G$-инвариантной вероятностной меры на обволакивающей группе обеспечивается следующим аналогом теоремы Рыль-Нарджевского о неподвижной точке [3].

Лемма. Пусть группа $G$ действует линейно и дистально по отношению к слабой сходимости на слабо компактном выпуклом мнодестве вероятностных мер. Тогда это действие имеет неподвижную точку.

Инвариантное интегрирование по построенной мере позволяет усреднить метрику, индуцированную на $G$ произвольной метрикой пространства $E(G, K)$, и рассмотреть $G$ как группу изометрий соответствующего пополнения $\bar{G}$.

Действие $G$ на банаховом пространстве непрерывных функций на $\bar{G}$ является почти-периодическим, и упомянутая выше лемма позволяет вложить $G$ в топологическую компактную группу $\widehat{G}$. При этом свойство отсутствия малых орбит влечет относительное NSS-свойство для вложения $G \subset \widehat{G}$.

Полученный результат можно использовать как признак линейной представимости групп. Подобные методы можно использовать также для исследования вопроса о финитной аппроксимируемости конечно-порожденных групп в тех случаях, когда непосредственное построение достаточной системы подгрупп конечного индекса затруднительно. Доказательство приводимого нами следствия использует теорему Мальцева об аппроксимации.

СлЕДСтвИЕ. Конечно-порожденная группа финитно аппроксимируема тогда и только тогда, когда она әффективно и дистально действует на некотором компакте.

Условие конечной порожденности рассматриваемых групп используется при доказательстве метризуемости пространств, возникающих в процессе доказательства основной теоремы. При этом используется следующий метризационный принцип.

ЛЕмма. Пусть конечно-порожденная группа $G$ действует гомеоморфизмами на компактах $K_{\alpha}, \alpha \in A$, и пусть $*$ - точка произведения $\prod_{\alpha \in A} K_{\alpha}$. Тогда замькание орбиты $G *$ метризуемо.

СлЕДСтвиЕ. Группа $G$, допускающая әффективное дистальное действие без мальх орбит на некотором компакте, является линейной, т.е. допускает точное матричное представление над некоторым полем $\Delta$.

Последнее следствие вытекает из локальной теоремы Мальцева. В качестве поля $\Delta$ можно взять некоторую ультрастепень поля комплексных чисел.

\section{СПИСОК ЛИТЕРАТУРЫ}

[1] Мерзляков Ю. И. Рациональные группы. М.: Наука, 1987. [2] Бронштейн И. У. Расширения минимальных групп преобразований. Кишинев: Штиинца, 1975. [3] Ryll-Nardzewski C. // Proc. of the Fifth Berkeley Symp. on Math. Statistics and Probability. V. 2. Berkeley: Univ. of California, 1967. P. 55-61.

Московский государственный

Принято редколлегией университет им. М. В. Ломоносова

07.05.1999 